\title{
Trump als Symptom: Populistische Schockpolitik und die Krise der Demokratie
}

\author{
Simon Schleusener
}

\section{Nothing's Shocking}

Die Wahl Donald Trumps zum 45. Präsidenten der USA hat weite Teile der politischen Öffentlichkeit in eine Art Schockzustand versetzt. Nicht nur hatte kaum jemand ernsthaft damit gerechnet, dass sich der populistische Immobilienmogul und TV-Entertainer gegen eine versierte Politikerin wie Hillary Clinton (immerhin ehemalige First Lady, Außenministerin und Senatorin für den Bundesstaat New York) durchsetzen würde; auch scheint sich seit der Wahl zu zeigen, dass es die Trump-Regierung ,ernst meint ${ }^{6}$, d. h. noch rücksichtsloser mit der politischen Opposition und kritischen Medien, staatlichen Institutionen und der Justiz, internationalen Abkommen und Allianzen sowie den allgemeinen Gepflogenheiten, Normen und Traditionen des politischen Geschäfts verfährt, als man dies ohnehin befürchtet hatte. And what's gonna happen next? In der Tat herrscht vielerorts der Eindruck vor, die USA hätten sich seit dem 8. November 2016 in ein gänzlich fremdes Land verwandelt.

Ziel dieses Aufsatzes ist es, den beschriebenen Schockzustand mit Blick auf seine beiden Seiten zu analysieren: einerseits hinsichtlich der von Trump betriebenen Politik der Störung ${ }^{1}$ und des Schocks (vgl. Klein 2017), ihrer Techniken und Taktiken, Voraussetzungen und Verfahrensweisen; andererseits aber

\footnotetext{
${ }^{1}$ Hier sei darauf hingewiesen, ,,dass Störungen nicht als autonome Entitäten, sondern immer nur in einer perspektivischen Relation existieren“ (Koch/Nanz 2014: 96). Was folglich von der liberalen Kommentatorin als Störung der politischen Anstandsregeln und des demokratischen Prozesses begriffen wird, mag vom Trump-Anhänger vielmehr als Wiederherstellung einer Ordnung verstanden werden, die während der Präsidentschaft Obamas abhandengekommen ist.
}

\footnotetext{
S. Schleusener $(\square)$

Berlin, Deutschland

E-Mail: simon.schleusener@fu-berlin.de
} 
auch mit Blick auf die geschockte (liberale) Öffentlichkeit und dasjenige, was sich in loser Anlehnung an Wolfgang Hagen als ihre ,Gegenwartsvergessenheit ${ }^{*}$ bezeichnen lässt (vgl. Hagen 2003). Der Aufstieg Trumps von der Marke zum Präsidenten ist freilich nicht voraussetzungslos, sondern hat sich auf vielfältige Weise angekündigt. So hat sich während der Präsidentschaft Obamas - dessen Wahl gerade in Europa als Triumph des ,guten', weltoffenen, multikulturellen und liberalen Amerikas gedeutet wurde - ein gänzlich anderes Amerika konstituiert, das lange unterhalb der medialen Aufmerksamkeitsschwelle verblieb und auch von den Demoskopen nicht adäquat erfasst wurde. Dieses andere Amerika (,the deplorable America', um es in Anlehnung an Hillary Clinton zu sagen ${ }^{2}$ ) lässt sich als Ausdruck eines signifikanten Rechtsrucks verstehen, vor allem aber markiert es eine Krise des klassischen Establishments, des westlichen Modells der liberalen Demokratie und ihrer lange als alternativlos geltenden ökonomischen Grundsätze (Neoliberalismus, Freihandel, Globalisierung). Wenn Trump im vorliegenden Aufsatz als Symptom begriffen werden soll, dann heißt dies in erster Linie, den Ausgang der Wahl 2016 nicht als isoliertes Phänomen zu betrachten, sondern ihn im Kontext dieses ,populistischen Moments ‘ zu verorten, der aktuell die politische Ordnung Europas und der USA - und mithin der Welt insgesamt - herausfordert und durcheinanderbringt. ${ }^{3}$ Ferner heißt es, anzuerkennen, dass die viel zitierte ,Krise der Demokratie keinesfalls erst durch die Wahl Trumps, den Brexit und den Aufstieg des Rechtspopulismus ausgelöst wurde, sondern vielmehr als Ursache dieser Entwicklungen zu begreifen ist. ${ }^{4}$ „Der Populismus“, so Jörke und Selk, ,ist eine Reaktion auf die nicht eingehaltenen Versprechen der Demokratie“ (Jörke/Selk 2017: 13).

Dass Trumps Präsidentschaft von zahlreichen Problemen und Konflikten geprägt ist, sollte nicht vorschnell als Hinweis gedeutet werden, der ,Spuk könne schon bald wieder vorüber sein. Sicherlich: Angesichts der fortwährenden Turbulenzen (die sog. ,Russia investigation', Stormy Daniels und andere private Affären, Konflikte mit dem FBI und dem Justice Department, ständige Personalwechsel und parteiinterne Flügelkämpfe ${ }^{5}$ ) ist es durchaus möglich, dass Trumps Amtszeit schon vor der nächsten regulären Präsidentschaftswahl der Geschichte angehört. Die über $40 \%$ der US-Wahlberechtigten, die ihn den meisten Statistiken zufolge kontinuierlich unterstützen (vgl. RealClearPolitics 2018), werden jedoch nicht annähernd so schnell wieder verschwunden sein. Um das Phänomen (oder besser:

\footnotetext{
${ }^{2}$ Vgl. hierzu Metha (2016) und Schleusener (2018: $30 \mathrm{f}$.).

${ }^{3} \mathrm{Vgl}$. Mouffe (2018: 11): ,We can speak of a ,populist moment' when, under the pressure of political or socioeconomic transformations, the dominant hegemony is being destabilized by the multiplication of unsatisfied demands. [...] This, I contend, is precisely what characterizes our present conjuncture.“

${ }^{4}$ Verwiesen sei hier auf Phänomene, die unter den Stichworten ,Postpolitik', ,Postdemokratie“ oder ,Politik der Alternativlosigkeit' untersucht worden sind. Vgl. etwa Mouffe (2005); Crouch (2008); Fisher (2009); Agamben/Badiou/Bensaïd u. a. (2012) sowie Lammert/Vormann (2017).

${ }^{5} \mathrm{Zu}$ den andauernden Turbulenzen im Weißen Haus, vgl. u. a. die ,Enthüllungsbücher“ von Michael Wolff und Bob Woodward (Wolff 2018 und Woodward 2018).
} 
das Ereignis) Trump zu begreifen, ist es somit notwendig, über die Person Trump hinauszugehen und die ökonomischen, politischen, kulturellen, medialen und ideologisch-affektiven Bedingungen und Transformationen in den Blick zu nehmen, die seinen Aufstieg überhaupt erst ermöglicht haben. ${ }^{6}$

Wie eingangs beschrieben, ist die Wahl Trumps von der liberalen Öffentlichkeit mehrheitlich als Schock empfunden worden: quasi als ,Einbruch des Realen', wie sich in Anspielung an Trumps Twitter-Account (@realDonaldTrump) formulieren lässt. Besser als an die Psychoanalyse Lacans - der das Reale im Sinne eines unartikulierbaren Alptraums oder Traumas konzipiert - lässt sich in diesem Zusammenhang jedoch an die Filmphilosophie von Gilles Deleuze anknüpfen. Die Analogie zum Kino ist dabei nicht ganz zufällig, denn in der Tat kann das politische Agieren der Trump-Regierung mitunter wie eine Mischung aus Reality TV und kinematografischem Spektakel erscheinen: als Film, der in Echtzeit abläuft, ohne dass für das staunende Publikum die Möglichkeit eines Eingriffs oder einer Intervention besteht. Auf in mancher Hinsicht vergleichbare Weise hat Deleuze den Übergang des ,Bewegungs-Bilds ' zum ,Zeit-Bild' beschrieben, nämlich durch das Auftauchen von kinematografischen Figuren, die eher ,Sehende` als ,Handelnde` sind, da sie vermehrt in ,rein optische Situationen ' geraten, die jeglicher Handlungsmöglichkeit entzogen scheinen. Im italienischen Neorealismus etwa wird die Filmfigur

„selbst gewissermaßen zum Zuschauer. Sie bewegt sich vergebens, rennt vergebens und hetzt sich vergebens ab, insofern die Situation, in der sie sich befindet, in jeder Hinsicht ihre motorischen Fähigkeiten übersteigt und sie dasjenige sehen und verstehen läßt, was nicht mehr von einer Antwort oder Handlung abhängt. Kaum zur Reaktion fähig, registriert sie nur noch. Kaum zum Eingriff in eine Handlung fähig, ist sie einer Vision ausgeliefert, wird von ihr verfolgt oder verfolgt sie selbst“ (Deleuze 1997a: 13).

Dasjenige, was hier gesehen wird, löst gewissermaßen einen Schock aus und demobilisiert. Laut Deleuze kann dieser Zusammenbruch des „sensomotorischen Schemas“ (ebd.: 60) jedoch auch zu neuen Reflexionsweisen und folglich zu einer Änderung des Habitus führen. ${ }^{7}$ Es wäre zu hoffen, dass das Ereignis Trump der ,reale“ Donald Trump - einen ähnlichen Effekt auf die schockierte politische Öffentlichkeit haben wird. Dass also der Schock nicht zum Dauerzustand wird, sondern letztlich eine heilsame Wirkung erzielt, indem er das Denken affiziert und in Bewegung versetzt, sodass sich auf diese Weise neue Handlungsoptionen ergeben.

\footnotetext{
${ }^{6}$ Trump auf diese Weise ,symptomatisch' zu lesen, hat folglich wenig mit der literaturwissenschaftlichen Diskussion um symptomatic reading und surface reading zu tun (vgl. Best/Marcus 2009). Denn eher als um das Verhältnis von Oberfläche und Tiefe geht es darum, einen Schritt zurückzugehen, um so die diversen Relationen zu erfassen, in die der Trumpismus eingebunden ist - und die ihn derart wuchern lassen.

${ }^{7}$ Vgl. hierzu auch Deleuzes Anmerkungen über die ,dritte Synthese der Zeit" in Differenz und Wiederholung (Deleuze 1997b: 118-130 und Schleusener 2015: 87-96).
} 


\section{Politik der Akzeleration}

Das Gefühl der Ohnmacht und des Schocks bezieht sich nicht allein auf den ,Inhalt‘ von Trumps Politik, etwa darauf, wie sein rechtspopulistischer Nativismus den Status quo des transnationalen politisch-ökonomischen Gefüges bedroht. Auch ist es nicht bloß die allgemeine ,Form', d. h. die ständige Verletzung der politischen Anstandsregeln oder die Umkehrung des Verhältnisses von Fakt und Fiktion, truth and fake (vgl. Schleusener 2018b). Sicherlich spielt all dies eine Rolle. Noch wesentlicher ist allerdings die zeitliche Komponente: der Eindruck also, dass sich die Ereignisse überschlagen, dass man stets überrumpelt wird. Dieses Phänomen lässt sich durchaus im Sinne der ,dromologischen“ Überlegungen Paul Virilios zum Verhältnis von Geschwindigkeit und Politik (Virilio 1980) analysieren. Wie Virilio argumentiert, stellt die Geschwindigkeit nicht nur das bestimmende Element der modernen Gesellschaft dar, sondern erweist sich auch als entscheidender Faktor für die Durchsetzung militärischer, politischer oder ökonomischer Ziele. ${ }^{8}$ Was Virilio diesbezüglich über die Raketentechnologie erläutert - „,der Unmittelbarkeit der Aktion über große Distanzen korrespondiert die Niederlage des überraschten Gegners“ (ebd.: 177) - lässt sich auf vergleichbare Weise über die „absolute Geschwindigkeit“ digitaler Übertragungsmedien sagen (Virilio 1993: 68).

Wesentlich für Trumps Politik der Geschwindigkeit ist in dieser Hinsicht das Echtzeitmedium Twitter, durch das es ihm gelingt, seine über 59 Mio. Follower jederzeit in Sekundenschnelle zu erreichen (vgl. Turner 2018 und Cowls/Schroeder 2018). Seit seiner Amtseinführung im Januar 2017 hat Trump ca. 7000 Tweets abgesetzt, das entspricht einer Rate von annähernd neun Tweets pro Tag (vgl. Trump Twitter Archive 2018). Jeder dieser Tweets eröffnet potenziell einen neuen Schauplatz im medialen Diskurs oder führt zu einer Umformung der öffentlichen Aufmerksamkeitsökonomie. Etwa kann ein Beitrag des konservativen Fernsehsenders Fox News unmittelbar zu einem Tweet animieren, der unter Umständen eine internationale Krise auslöst, zugleich aber Trumps base in Ekstase versetzt. Die ,Unmittelbarkeit" des Mediums fördert mithin die Vorstellung, es existiere ein direkter Draht zwischen Trump und seinen Followern. So dient Twitter dem am Reality TV geschulten Präsidenten nicht bloß dazu, seine vermeintliche Authentizität, Nahbarkeit und Impulsivität zu unterstreichen (ein wesentlicher Baustein seiner ,charismatischen“ Amtsführung); auch stellt das Medium ein perfektes Instrument zur affektiven Mobilisierung seiner Anhänger*innen dar und dient der Beschäftigung und Ablenkung der kritischen Medien. Die von Fred Turner aufgeworfene Frage - „How a Medium Designed for Democracy Became an Authoritarian's Mouthpiece“ (Turner 2018) - ist daher irreführend. Bei Twitter handelt es sich um kein ursprünglich demokratisches Medium, das Trump für seine Zwecke umfunktioniert hat. Vielmehr fungiert die auf maximal 280 (vormals 140) Zeichen begrenzte Twitter-Mitteilung als ideales Medium für die rechtspopulistische Strategie der Provokation und Zuspitzung: „Sie darf nicht nur, sie muss verkürzen,

\footnotetext{
${ }^{8}$ Vgl. Virilio (1980: 63): „Der abendländische Mensch erschien trotz einer weniger zahlreichen Bevölkerung als überlegen und dominierend, weil er schneller war.“
} 
zuspitzen, personalisieren“ (Seeßlen 2017: 73 f.). Bei Twitter handelt es sich buchstäblich um ,Gezwitscher", d. h. um ein post-diskursives Signal-Medium, das sich für die affektive Mobilmachung der eigenen Anhänger*innenschaft ebenso gut eignet wie für die Provokation des politischen Gegners.

Trumps akzelerationistische Twitter-Politik fördert somit einerseits das Vergessen und Verblassen: Jeder Skandal wird stets durch den nächsten abgelöst, auf jeden Schlag folgt ein unmittelbarer Gegenschlag. ${ }^{9}$ Was daher entsteht, ist der Eindruck einer allgemeinen Zerstreuung. Immer scheint die Zeit zu fehlen, sich auf die vielen kleinen Skandale zu konzentrieren, da diese jeweils von der nächsten Eilmeldung, dem nächsten Ablenkungsmanöver oder Gegenschlag, durchgestrichen werden. Vergleichbar mit der Argumentation Virilios korrespondiert mit der Politik der Geschwindigkeit also auch hier eine Logik - und , ̈̈sthetik ‘ - des Verschwindens (vgl. Virilio 1986).

Andererseits führt Trumps Beschleunigungspolitik auch zum Anwachsen der Kontingenz. Die Aufmerksamkeit richtet sich stets auf das Kommende, das jedoch umso weniger planbar erscheint: „What will Trump say next?“10 Aus genau diesem Grund ist es (abgesehen vom reinen Tagesjournalismus) auch so schwierig, über Trump zu schreiben. Der vorliegende Text etwa entstand größtenteils im Zeitraum von September bis Oktober 2018, also noch vor den Zwischenwahlen im November. Derzeit ist kaum absehbar, was sich ereignet haben wird, wenn der Aufsatz veröffentlicht ist. Verlieren die Republikaner ihre Mehrheit im Kongress? Feuert Trump seinen Attorney General? Kippt er das 14. Amendment? Fördert Robert Mueller neue Indizien gegen Trump und seine Alliierten zutage? Oder wird Mueller abgesetzt? Kommt es gar zum Amtsenthebungsverfahren? Und was passiert auf internationaler Ebene: Beendet Nordkorea sein Atomprogramm oder kommt es zur neuerlichen Eskalation? Was geschieht mit Syrien? Was wird aus der NATO und den internationalen Freihandelsabkommen? Dadurch, dass Trump den Status quo aufkündigt, der die amerikanische Politik in den letzten Jahrzehnten bestimmt hat, verflüchtigt sich auch jedes Gefühl der Sicherheit und Erwartbarkeit, das sich während der langen Jahre politischer Alternativlosigkeit noch einstellen konnte. Alles scheint wieder möglich - auch das Schlimmste. Die potenziellen Konsequenzen dieser Politik, das mögliche aus-dem-Ruder-Laufen

\footnotetext{
${ }^{9}$ Man denke etwa an Trumps Umgang mit der Veröffentlichung des Access-Hollywood-Tape im Oktober 2016. Allgemein wurde damit gerechnet, dass die Aufnahme (,grab them by the pussy ${ }^{\circ}$ usw.) Trumps Präsidentschaftsambitionen zunichtemachen würde. Trump reagierte jedoch, indem er seine misogynen Äußerungen herunterspielte (,this was locker room talk') und Hillary Clinton der Komplizenschaft mit den sexuellen Übergriffen ihres Ehemanns bezichtigte. So ließ er auf einer spontan einberufenen Pressekonferenz - direkt vor dem zweiten Rededuell mit seiner Kontrahentin - mehrere vermeintliche Opfer von Ex-Präsident Bill Clinton zu Wort kommen.

${ }^{10}$ Vgl. Avanessian/Hennig (2018: 171): „Beim aufmerksamen Konsum von Massenmedien fällt auf, dass sie sich immer weniger auf Schon-Geschehenes oder Gerade-Geschehendes, sondern auf eine als Bedrohung geschilderte Zukunft orientieren: Was wird ,jetzt ' (also in der die Gegenwart bestimmenden Zukunft) geschehen? What will Trump say next? Wann wird sich der nächste Terrorangriff ereignen? Wo wird entschieden werden, was zu tun ist?" Zur Transformation der Zukunft, die heute kaum noch als Möglichkeitsraum, sondern zunehmend als bedrohlich und katastrophisch gedacht wird, vgl. auch Horn (2014) sowie Koch/Nanz/Pause (2016).
} 
oder Abgleiten ins Chaos, macht Trump sich durchaus zunutze, etwa indem er die internationalen ,Partner' zu Zugeständnissen zwingt, um eben jenes Schlimmste $\mathrm{zu}$ verhindern (eine Strategie, die nicht selten erfolgreich ist, denn wie das globale Bankensystem gelten auch die USA als , too big to fail' $)$. Es ist klar, dass diese Politik der kalkulierten Überrumpelung ganz wesentlich von der Nutzbarmachung der Medien abhängt: von der Schnelligkeit und Unmittelbarkeit der social media platforms; der Möglichkeit, gezielt Falschmeldungen und Gerüchte zu streuen (Twitter, Facebook, Breitbart News); dem Bedienen der ,Sensationslust' aufseiten der traditionellen Nachrichtenmedien (Presse und Fernsehen).

So ist das vielleicht größte Paradox Trumps, dass einerseits zwar seine gesamte politische Existenz medial bedingt ist, d. h. ohne ausgeprägte Medienaffinität gar nicht denkbar wäre, ${ }^{11}$ dass andererseits aber die Verachtung ,der Medien " (,the dishonest media“, ,enemy of the people“, „fake news“, „failing New York Times“ usw.) zum Grundrepertoire seines Populismus gehört.

\section{Race/Class}

Im medialen und akademischen Diskurs konkurrieren im Wesentlichen zwei unterschiedliche Narrative, die den Ausgang der Präsidentschaftswahl 2016 erklären sollen. Zum einen existiert die Lesart, dass die Wahl Trumps unmittelbar rassistisch (und teilweise sexistisch ${ }^{12}$ ) motiviert war. Nach acht Jahren unter einem afro-amerikanischen Präsidenten, so die These, hat sich ein regelrechter whitelash ereignet (vgl. Agerholm 2016). Trumps systematische Mobilisierung xenophober, rassistischer, islamfeindlicher und misogyner Affekte wird dementsprechend so gedeutet, dass sie der verbreiteten Sehnsucht nach Wiederherstellung der Ordnung weißer, männlicher Vorherrschaft (,Make America great again“) entgegenkommt - einem Impuls, der sich mit Zygmunt Bauman als ,retrotopisch“ bezeichnen lässt (Bauman 2017). ${ }^{13}$ Die andere Lesart ist stärker ökonomisch fundiert und verweist darauf, dass durch die zunehmende Fokussierung der Demokraten (und allgemein der

\footnotetext{
${ }^{11}$ Die mediale Bedingtheit von Trumps Populismus lässt sich freilich nicht nur am Einsatz von Twitter und anderer sozialer Medien festmachen. Auch lässt sich argumentieren, dass Trump ohne sein medial produziertes Erfolgsimage und die Popularität, die er als Moderator der Reality Show The Apprentice erlangte, niemals hätte Präsident werden können (vgl. Keefe 2019). Denn einerseits profitierte er zum Zeitpunkt seiner Kandidatur von einem Bekanntheitsgrad, der den der meisten seiner republikanischen Konkurrenten deutlich in den Schatten stellte. Andererseits stürzten sich die Medien im Vorwahlkampf begierig auf die, TV-Star-Goes-Presidential-Candidate'-Story, was Trump ein wesentlich höheres Maß an Sendezeit bescherte.

${ }^{12} \mathrm{Vgl}$. etwa Trumps Invektiven gegen Hillary Clinton, die nicht nur als Symbol des liberalen Establishments, sondern auch als ,nasty woman' ohne ,stamina' attackiert wurde (vgl. Woolf 2016).

${ }^{13} \mathrm{Als}$,Retrotopien“ versteht Bauman „Visionen, die sich anders als ihre [utopischen] Vorläufer nicht mehr aus einer noch ausstehenden und deshalb inexistenten Zukunft speisen, sondern aus der verlorenen/geraubten/verwaisten, jedenfalls untoten Vergangenheit“"(Bauman 2017: 13).
} 
amerikanischen ,Linken“14) auf Themen wie Identität und Geschlecht, Diversität und Diskriminierung die Frage der Klassenzugehörigkeit und der sozial-ökonomischen Ungleichheit vernachlässigt wurde, was sich Trumps auf die white working class zugeschnittener Wahlkampf zunutze machte. Unterstützt wird diese Lesart dadurch, dass sich (mit Ausnahme Floridas) alle derjenigen Bundesstaaten, die 2016 von den Republikanern hinzugewonnen wurden (Iowa, Wisconsin, Michigan, Ohio und Pennsylvania), in der sog. rust belt area befinden - in einer Gegend also, die dezidiert durch die Industriearbeiterschaft geprägt ist und traditionell eher von den Demokraten dominiert wird. ${ }^{15}$

Die tendenzielle ,Konkurrenz ' dieser beiden Interpretationen verweist in erster Linie auf die jüngere Entwicklungsgeschichte der Linken und des kritischen Diskurses, der im Zuge der Postmodernisierung (und ,Kulturalisierung') der Geistes- und Sozialwissenschaften in den 1980er und 1990er Jahren zunehmend vom marxistisch geprägten Theoriehorizont der Vergangenheit abrückte und sich bisweilen mit Ansätzen wie der ,Politik der Anerkennung 'verbündete (vgl. Taylor 1992; Fraser/Honneth 2003). ${ }^{16}$ Seit der Wirtschafts- und Finanzkrise von 2008 ist jedoch ein gewisses Comeback marxistischer und anderer kapitalismustheoretischer Perspektiven zu beobachten, sodass gerade auch das Phänomenon des Populismus heute vermehrt im Kontext des Ökonomischen analysiert wird. ${ }^{17}$

Natürlich lässt sich für das Verständnis der Wahl Trumps zunächst an beide Lesarten anknüpfen. So ist unbestritten, dass Rassismus in den USA - die sich zu wesentlichen Teilen noch immer als räumlich und sozial segregierte Nation manifestieren - längst nicht der Vergangenheit angehört, und dass sich alle mit Obamas

\footnotetext{
${ }^{14}$ Dass der Begriff der Linken hier in Anführungszeichen steht, soll nicht dessen generelle Brauchbarkeit infrage stellen, sondern darauf hinweisen, dass der Terminus in den USA zuweilen anders verwendet wird. Beispielsweise werden selbst moderate Forderungen nach gewissen sozialen Mindeststandards (allgemeine Krankenversicherung, Lohnfortzahlung im Krankheitsfall, Mutterschutz) von konservativer Seite als ,left-wing' oder ,socialist" etikettiert. Zudem existiert eine grundsätzliche Vermischung mit dem Liberalismus (,left" und ,liberal ' werden nicht selten synonym verwendet), sodass liberale Forderungen, die sich etwa gegen Diskriminierung richten, in der Regel auch als , leftist' gelten. In Abgrenzung zum Mainstream-Liberalismus der Demokratischen Partei bezeichnen sich Demokraten mit dezidiert linker Haltung heute mehrheitlich als , progressives".

${ }^{15}$ Zur Relevanz der Klassenthematik im US-Präsidentschaftswahlkampf 2016 (und allgemein für den Aufstieg des Rechtspopulismus), vgl. Schleusener (2018a).

${ }^{16}$ Autoren wie Walter Benn Michaels haben derlei ,identitätspolitische‘ Ansätze explizit mit der Logik des Neoliberalismus in Verbindung gebracht und ihnen Klassenblindheit und Ignoranz gegenüber der ökonomischen Dimension von Ungleichheit vorgeworfen (vgl. Michaels 2006). Mittlerweile existieren allerdings eine Reihe von Versuchen der Vermittlung zwischen ,race'- und ,class'-basierten Konzeptionen, die sich gegen die dominante Spielart der identity politics richten (vgl. Haider 2018).

${ }^{17}$ Als Referenz hierfür dient u. a. das Werk Polanyis, der das Aufkommen des Faschismus im Kontext der Krise des Laissez-Faire-Kapitalismus erklärt hatte: „In order to comprehend German fascism, we must revert to Ricardian England“ (Polanyi 2001: 32). Vgl. auch - mit Blick auf den heutigen Populismus - Holmes (2018). Zur ökonomischen Dimension des Populismus siehe außerdem Stegemann (2017); Jörke/Selk (2017); Mouffe (2018) und Manow (2018).
} 
Wahl verbundenen liberalen Hoffnungen bezüglich eines ,Post-racial America“ (Schorr 2008) heute als naiv darstellen. Trump hat sich die konfliktreichen Rassenbeziehungen, soziale Stereotypen und kulturell codierte Identitätskonstruktionen politisch zunutze gemacht und dabei mehrfach die Grenze überschritten, die traditionell zwischen dem Mainstream-Konservatismus der Republikaner und dem offenen Rassismus der white supremacists gezogen wird. ${ }^{18}$ Typisch für die Strategie der neuen Rechten ist dabei, dass Trump den Rassismusvorwurf freilich stets von sich weist (,I'm the least racist person you've ever interviewed ') und das Überschreiten ,roter Linien“ der politischen Korrektheit mitunter als Spiel begriffen wird, das in erster Linie auf die (Über-)Reaktion der etablierten Medien und der liberalen Öffentlichkeit gemünzt ist (vgl. Strick 2018). Unabhängig von Trumps, wirklicher" Haltung gegenüber Rassendiskriminierung und ethnischer Differenz ist jedoch allzu offensichtlich, dass sich Rechtsextreme, weiße Rassisten und die Alt-Right-Bewegung seit Trumps Wahl zum Präsidenten im Aufwind fühlen.

Schaut man sich den Ausgang der Wahl 2016 allerdings genauer an, dann wird deutlich, dass es sich eine vornehmlich den ,Race'-Aspekt betonende Lesart zu einfach macht. Hier ist zunächst zur Kenntnis zu nehmen, dass die Demokratin Hillary Clinton insgesamt fast drei Millionen Stimmen mehr erhielt als Donald Trump. Wenn also von einem ,massiven Rechtsruck' die Rede ist, dann betrifft dies in erster Linie die Entwicklung innerhalb der Republikanischen Partei, die seit dem Aufkommen der Tea-Party-Bewegung kontinuierlich nach rechts gedriftet ist. Dass Trump trotz seines deutlichen Stimmendefizits zum Präsidenten gekürt wurde, verdankt sich indes einer Besonderheit des amerikanischen Wahlsystems: nämlich der Tatsache, dass für die Wahl des Präsidenten nicht die popular vote (d. h. die Anzahl der abgegebenen Stimmen), sondern das electoral college ausschlaggebend ist, das sich aus den von den Einzelstaaten entsendeten Wahlmännern zusammensetzt. Da die Anzahl der Wahlleute die Einwohnerzahl des jeweiligen Bundesstaats nur bedingt widerspiegelt, sind die bevölkerungsreichen Staaten - unter denen viele zu den Demokraten tendieren (Kalifornien, New York usw.) - einem gewissen Nachteil ausgesetzt. Vor diesem Hintergrund lässt sich erklären, dass die Republikaner seit 1992 zwar nur bei einer Wahl die popular vote gewannen (2004 durch George W. Bush), aber drei Mal den Präsidenten stellten (2000, 2004, 2016). ${ }^{19}$ In Anlehnung an Trump lässt sich somit behaupten: The system is indeed rigged (but it is rigged in favor of the GOP...).

\footnotetext{
${ }^{18}$ Vgl. etwa Trumps schon lange vor seiner Kandidatur geäußerte Behauptung, Obama sei kein amerikanischer Staatsbürger (,he doesn't have a birth certificate') oder seine Aussagen über einen Aufmarsch von white supremacists in Charlottesville (,very fine people, on both sides ').

${ }^{19}$ Hinzu kommt, dass jeder Bundesstaat - vollkommen unabhängig von seiner Einwohnerzahl - durch jeweils zwei Senatoren vertreten wird, was die einwohnerstarken Staaten erneut benachteiligt. Für die Demokraten ist es dementsprechend besonders schwierig, die Mehrheit im Senat zu erringen, dessen Zusammensetzung nicht dem Gesamtverhältnis der abgegebenen Stimmen entspricht, sondern auf einer disproportionalen Verteilung zugunsten der gering bevölkerten (und meist ländlich geprägten) Bundesstaaten beruht.
} 


\begin{tabular}{|c|c|c|c|c|}
\hline White & $37 \%$ & $58 \%$ & \multirow[b]{2}{*}{$88 \%$} & \\
\hline Black & & & & $8 \%$ \\
\hline Hispanic/Latino & & $65 \%$ & $29 \%$ & \\
\hline Asian & & $65 \%$ & $29 \%$ & \\
\hline Other & & $56 \%$ & & \\
\hline
\end{tabular}

Abb. 1 Exit polls der Präsidentschaftswahl 2016 (,Race'). Links die Prozentpunkte für die demokratische Kandidatin (Hillary Clinton), rechts für den republikanischen Bewerber (Donald Trump)

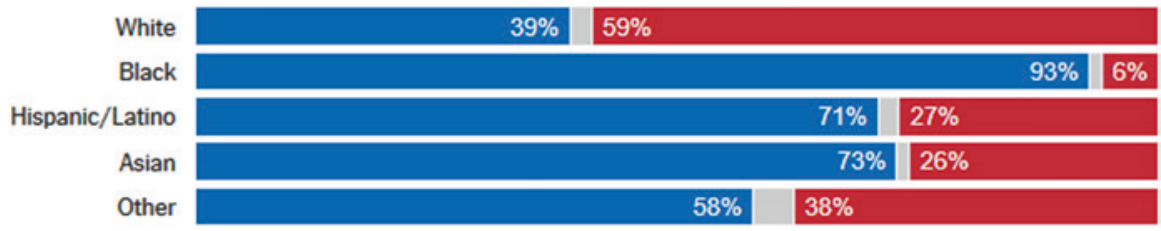

Abb. 2 Exit polls der Präsidentschaftswahl 2012 (,Race'). Links die Prozentpunkte für den demokratischen Kandidaten (Barack Obama), rechts für den republikanischen Bewerber (Mitt Romney)

Betrachtet man außerdem die im Rahmen der exit polls ermittelten demografischen Daten (vgl. auch Schleusener 2018a: 24 f.), dann fällt auf, dass weiße Wähler*innen ironischerweise die einzige ethnische Gruppe darstellen, die 2016 zu einem geringeren Prozentsatz die Republikaner gewählt hat als vier Jahre zuvor (58 \% gegenüber $59 \%$ ). Dagegen konnte Trump (im Vergleich zu Romney 2012) bei Afro-Amerikaner*innen, Asiat*innen sowie Latinos und Latinas leicht zulegen (vgl. Abb. 1 und 2). Am auffälligsten jedoch ist die Wählerwanderung im Bereich der einkommensschwachen Wählergruppen, d. h. bei Personen mit einem Jahreseinkommen, das unterhalb von 30.000 bzw. 50.000 US\$ liegt. Zwar hat Hillary Clinton hier noch einen recht deutlichen Vorsprung vor Trump - denn traditionell tendieren Wähler*innen aus den unteren Einkommensschichten zu den Demokraten -, doch ist es den Republikanern genau hier am besten gelungen, Stimmen hinzuzugewinnen, während die Demokraten hohe Verluste hinnehmen mussten (vgl. Abb. 3 und 4). In dieser Hinsicht lässt sich durchaus argumentieren, dass die ökonomische Dimension für die Wahl Trumps eine entscheidende Rolle gespielt hat. ${ }^{20}$

\footnotetext{
${ }^{20}$ Zur Unterstützung des Arguments, dass ,Race“ den wesentlichen Faktor für den Ausgang der Wahl 2016 darstellt, wurde mehrfach hervorgehoben, dass weiße Männer und Frauen fast sämtlicher Bildungsschichten, Alters- und Einkommensgruppen überwiegend Trump gewählt haben, während die Wähler*innen aller anderen ethnischen Gruppen mit deutlicher Mehrheit für Hillary Clinton stimmten (vgl. Henley 2016). Zwar verdeutlicht dies die allgemeine Relevanz ethnischer Differenz für die US-amerikanische Politik, doch ist die Dominanz der Republikaner unter WeiBen keine Neuigkeit. Laut den exit polls betrifft die wesentlichste Fluktuation, die sich zwischen 2012 und 2016 ereignet hat, vielmehr die Abwanderung vieler Wähler*innen aus den unteren Einkommensschichten von den Demokraten zu den Republikanern.
} 


\begin{tabular}{|r|r|r|r|}
\hline Under $\$ 30,000$ & \multicolumn{2}{|c|}{$53 \%$} & $41 \%$ \\
\cline { 2 - 4 }$\$ 30,000-\$ 49,999$ & \multicolumn{2}{|c|}{$51 \%$} & $42 \%$ \\
\hline $50,000-\$ 99,999$ & $46 \%$ & $50 \%$ \\
\hline $100,000-\$ 199,999$ & $47 \%$ & $48 \%$ \\
\hline $200,000-\$ 249,999$ & $48 \%$ & $49 \%$ \\
\hline 250,000 or more & $46 \%$ & $48 \%$ \\
\hline
\end{tabular}

Abb. 3 Exit polls der Präsidentschaftswahl 2016 (,Income`). Links die Prozentpunkte für die demokratische Kandidatin (Hillary Clinton), rechts für den republikanischen Bewerber (Donald Trump)

\begin{tabular}{|c|c|c|}
\hline Under $\$ 30,000$ & \multicolumn{2}{|r|}{$63 \%$} \\
\hline$\$ 30,000-\$ 49,999$ & & \begin{tabular}{l|l|}
$57 \%$ & $42 \%$ \\
\end{tabular} \\
\hline$\$ 50,000-\$ 99,999$ & $46 \%$ & $52 \%$ \\
\hline$\$ 100,000-\$ 199,999$ & $44 \%$ & $54 \%$ \\
\hline$\$ 200,000$ or more & $44 \%$ & $54 \%$ \\
\hline
\end{tabular}

Abb. 4 Exit polls der Präsidentschaftswahl 2012 (,Income`). Links die Prozentpunkte für den demokratischen Kandidaten (Barack Obama), rechts für den republikanischen Bewerber (Mitt Romney). Graphics from the article 'Election 2016: Exit Polls' by Jon Huang, Samuel Jacoby, Michael Strickland and K.K. Rebecca Lai originally appeared in the The New York Times on November 8, 2016 are copyright The New York Times and are used here by permission

\section{Die Krise des Neoliberalismus}

Wie aber ist es Trump gelungen, derart viele Wähler*innen aus der Arbeiterschicht und den unteren Einkommensgruppen an sich zu binden? Warum sind diese nicht (oder nicht mehr) für , linke ' Ideen empfänglich und wenden sich von den Demokraten ab? Wieso folgen sie ausgerechnet dem golfspielenden Milliardär und Geschäftsmann aus Queens, der letztlich dasselbe privilegierte Milieu repräsentiert wie die so arg verhasste, Elite'? In der Tat ist Trumps Image als ,Champion of the Working Class' einigermaßen erstaunlich - und dies nicht nur aufgrund seines Auftretens als knallharter businessman (in der Reality Show The Apprentice ebenso wie im ,wirklichen Leben'), sondern auch im Hinblick auf seine Wirtschaftspolitik, die erkennbar die top one percent und Besserverdienenden privilegiert. $^{21}$ Nichtsdestotrotz weist der Trumpismus gewisse

\footnotetext{
${ }^{21}$ Vgl. Klein (2017: 21): „Trump and his cabinet of former executives are remaking government at a startling pace to serve the interests of their own businesses, their former businesses, and their tax bracket as a whole. [...] He appointed his son-in-law, Jared Kushner, to head up a ,swat team “ stacked with corporate executives who have been tasked with finding new regulations to eliminate, new programs to privatize, and new ways to make the US government ,run like a great American company“.”
} 
Facetten auf, die ihm unter working class Americans eine erhebliche Popularität bescherten. Hierzu gehört einerseits Trumps Überschreitung der Grenzen politischer Korrektheit, seine Anti-Eliten- und Anti-Korruptions-Rhetorik (,Drain the Swamp!') sowie seine Rolle als politischer Outsider: sein direkter, unverblümter und als authentisch empfundener, politischer Stil'. Andererseits ist hervorzuheben, dass Trumps ökonomischer Nationalismus mit den Dogmen der Freihandelsdoktrin bricht, was seine Kandidatur - vergleichbar mit der von Bernie Sanders aufseiten der Demokraten - als Alternative zur neoliberalen Hegemonie erscheinen ließ, die lange als quasi alternativlos galt. So sprach sich Trump für die Einführung von Schutzzöllen aus und versprach, Arbeitsplätze zurück in die USA zu bringen. Zudem kritisierte er Freihandelsabkommen wie NAFTA, die seiner Auffassung nach $\mathrm{zu}$ einem massiven Outsourcing amerikanischer Jobs ins Ausland führten. In diesem Zusammenhang wird verständlich, weshalb die gigantische Mauer, die Trump an der Grenze zu Mexiko bauen lassen will, für seinen Wahlkampf eine derartige Relevanz hatte (und noch immer als Signatur des Trumpismus fungiert). Denn bei dem Projekt (unabhängig von seiner Realisierbarkeit, die mehr als fraglich ist) geht es nicht allein um den möglichen Schutz vor illegaler Einwanderung. Auch fungiert die Mauer als Metapher und Emblem für den Widerstand gegen eine Politik der ,offenen Grenzen` und des ökonomischen , Globalismus ‘.22

Was die Präsidentschaftswahl von 2016 somit auch verdeutlicht hat, ist eine veritable Krise des Neoliberalismus. ${ }^{23}$ Obwohl sich die globale Wirtschaft nach der Banken-, Finanz- und Eurokrise wieder einigermaßen erholt hat - weshalb vom mehrfach vorhergesagten ,,breakdown of the capitalist system“ (Wallerstein/ Collins/Mann u. a. 2013: 2) derzeit keine Rede sein kann -, lässt sich durchaus von einer anhaltenden ideologischen Krise der neoliberalen Doktrin sprechen, die heute von links wie von rechts attackiert wird. Dieses ideologische Unbehagen wurde im Wahlkampf nicht nur von Trump artikuliert, sondern mehr noch von Bernie Sanders, der in den Vorwahlen der Demokraten nur äußerst knapp (und unter Mithilfe des Partei-Establishments) gegen die deutlich favorisierte Hillary Clinton unterlag. Sowohl Trump als auch Sanders schienen eine Alternative zur neoliberalen Alternativlosigkeit zu verkörpern. Während Sanders jedoch für eine linke Alternative eintrat, die - für die USA erstaunlich - auf der Idee eines, demokratischen Sozialismus' und einem starken Sozialstaat fußte, war Trumps Alternative, basierend

\footnotetext{
${ }^{22}$ Zur kulturellen Semantik und Ikonografie der Mauer im Kontext rechtspopulistischer Affektpolitik, vgl. Koch (2019).

${ }^{23}$ Zur Krise des Neoliberalismus, vgl. Duménil/Lévy (2011); Wallerstein/Collins/Mann u. a. (2013); Mason (2015); Streeck (2016) und Schleusener (2018a). Aufgrund der neoprotektionistischen Tendenzen, die insbesondere von der Trump-Regierung befördert werden, stellt sich in der Tat die Frage, ob das aktuell dominante Akkumulationsregime (und sein Regulationsmodus) mit dem Begriff ,Neoliberalismus ' noch adäquat benannt ist. Vgl. Davies (2017: xviii): „The question inevitably arises, is this thing called ,neoliberalism“ now over? And if not, when might it be and how would we know?"
} 
auf dem nationalistischen Projekt eines protektionistischen Isolationismus, deutlich rechts konnotiert. Der ebenso simple wie anspielungsreiche Slogan dieses ökonomisch-nationalistischen Paradigmas lautet: ,America First! ${ }^{24}$

\section{Uncool Capitalism}

Einer Reihe von Autor*innen zufolge hat sich mit der Herausbildung des post-fordistischen Wirtschaftsmodells und dem Aufstieg des Neoliberalismus auch der gesamte kapitalistische ,Überbau' verändert. Laut Boltanski und Chiapello etwa wird der Kapitalismus schon lange nicht mehr im Sinne der protestantischen Arbeitsethik legitimiert, auf die sich noch Max Weber in seinen Arbeiten zum Verhältnis von Kultur und Ökonomie konzentrierte (vgl. Weber 1991). Vielmehr ist seit den 1960er und 1970er Jahren die Entstehung dessen zu beobachten, was Boltanski und Chiapello (im Anschluss an Weber) als ,neuen Geist des Kapitalismus ' bezeichnen: eine Ideologie, „die das Engagement für den Kapitalismus “ vor dem Hintergrund der veränderten Produktionsbedingungen und soziokulturellen Transformationen ",rechtfertigt" (Boltanski/Chiapello 2006: 43). ${ }^{25}$ Zentral an Boltanskis und Chiapellos Ansatz ist die Unterscheidung zwischen zwei unterschiedlichen Formen von Kapitalismuskritik (vgl. ebd.: 79-84), nämlich der ,Sozialkritik' (Kritik an der Ausbeutung von Arbeitskraft und der daraus resultierenden ökonomischen Ungleichheit) und der sog. ,Künstlerkritik' (Kritik an den Entfremdungs- und Entzauberungstendenzen des Kapitalismus, sein Hang zur Bürokratisierung und Rationalisierung im Sinne von Webers ,stahlhartem Gehäuse'). Was nun den neuen Geist des Kapitalismus auszeichnet, ist laut Boltanski und Chiapello die Tatsache, dass die historisch auf die Bohème zurückgehende Künstlerkritik zu weiten Teilen neutralisiert und für die Funktionsweise des Kapitalismus nutzbar gemacht worden ist. Diese Entwicklung lässt sich bereits in den 1960er Jahren beobachten, etwa mit Blick auf die Appropriation und Übernahme von Aspekten der Counterculture durch die damalige Konsumund Businesskultur (vgl. Frank 1998). Mehr noch gilt dies für die Zeit ab etwa 1990, ,„when] business became ,funky“, having shed its reputation for bureaucratic conformity“" (McGuigan 2009: 7). Die affektiv-ideologische Figuration dieser

\footnotetext{
${ }^{24}$ Die Parole ,America First ${ }^{*}$ hat im Kontext der US-amerikanischen Politik eine lange Geschichte und wurde vor Trump bereits von Isolationisten wie William Randolph Hearst, Paläokonservativen wie Pat Buchanan, Libertären wie Murray Rothbard oder Neonazis wie David Duke verwendet. Paradox an Trumps Aktualisierung des Slogans ist zweifellos die Tatsache, dass sich dieser nun insbesondere gegen die Liberalisierung des Welthandels richtet, die selbst ganz wesentlich auf Betreiben der USA zustande kam.

${ }^{25}$ In ihrer (hier deutlich verkürzt wiedergegebenen) Schematik gehen Boltanski und Chiapello im Wesentlichen von drei „historischen Etappen des kapitalistischen Geistes“ aus (vgl. Boltanski/ Chiapello 2006: 54-58). Gemeint ist 1) die auf den Unternehmer und Bourgeois zugeschnittene heroische Darstellungsweise, die sich im späten 19. Jahrhundert entwickelte; 2) der zwischen 1930 und 1960 auf das Großunternehmen mit gesellschaftlicher Verantwortung fokussierte und an die Massenproduktion des Konzernkapitalismus gekoppelte Modus; und schließlich 3) der ,neue Geist des Kapitalismus', der sich seit den 1960er Jahren vor dem Hintergrund der Globalisierung und der Entwicklung neuer Technologien herausgebildet hat.
} 
vermeintlich non-konformen, post-heroischen und auf der Grundlage fluider Netzwerke und ,flacher Hierarchien“ operierenden Variante des Kapitalismus hat Jim McGuigan als Cool Capitalism bezeichnet. ${ }^{26}$

Cool Capitalism, verstanden als neoliberalisierte Spielart des Weberschen Konzepts vom ,Geist des Kapitalismus ', bezieht seine kulturelle Kraft daher, dass „,the dreariness of business and private property, the dustiness of entrepreneurship" (Jameson 1992: 274) hier mit den Insignien der Gegenkultur und der Bohème versehen werden. Als idealtypische Verkörperung dieser Figuration, die heute eher im kalifornischen Silicon Valley als an der New Yorker Wall Street beheimatet ist, kann die Firma Apple (,Think Different") gelten, deren ehemaliger CEO Steve Jobs die perfekte Mélange aus Hippie und Kapitalist, Bohémien und Entrepreneur zur Darstellung brachte - eine kulturelle Performance, die noch heute in zahlreichen Werken der Populärkultur gewürdigt wird. ${ }^{27}$ Man ist Kapitalist, und gleichzeitig ist man es nicht; man ist der Chef des wertvollsten Unternehmens der Welt, und trotzdem gehört man niemals zum Establishment.

Der Kontrast zwischen dieser neoliberalen ,Coolness", die den neuen Geist des Kapitalismus artikuliert, und der von Trump verkörperten Retro-Ästhetik des successful businessman könnte, auf den ersten Blick betrachtet, kaum größer sein. Der sneakers tragende cool-kapitalistische Tech-Entrepreneur gibt sich nicht als Millionär, sondern als nerd zu erkennen. Trump dagegen protzt mit seinem Besitz und macht sich reicher als er wirklich ist (vgl. Greenberg 2018). In seiner TV-Serie The Apprentice wird gar nicht erst versucht, die Hierarchien und Ausschlussmechanismen des kapitalistischen Wettbewerbs zu verschleiern. Im Gegenteil ist die Popularität der Sendung gerade auf die Darstellung klassischer Autorität zurückzuführen (, You're fired!') - verbunden mit der Häme des Publikums für die losers, die den Anforderungen der televisualisierten Dog-Eat-Dog-Ökonomie nicht gewachsen sind. ${ }^{28}$ Wenn Trump in einem Interview erklärt: „Part of the beauty of me is that I'm very rich“ (vgl. King Jr. 2011), dann hyperrealisiert er gewissermaßen die alte Karikatur des Zigarre rauchenden Millionärs. ${ }^{29}$ Während die Logik

\footnotetext{
${ }^{26} \mathrm{Vgl}$. McGuigan (2009: 38): „Cool Capitalism is largely defined by the incorporation, and thereby neutralisation, of cultural criticism and anti-capitalism into the theory and practice of capitalism itself.“

${ }^{27}$ Siehe exemplarisch die Filmbiografie Jobs (2013) mit Ashton Kutcher in der Rolle des gleichnamigen Hippie-Unternehmers (vgl. Schleusener 2014: 320 f.).

${ }^{28} \mathrm{Vgl}$. Davis/Lukomnik/Pitt-Watson (2006: 205): „The show is presumably meant to be a metaphor of the business world: it's a place with many losers and few winners, a place where managers commonly get ahead by using cooperation as a mask for deception, disloyalty, and betrayal. [...] The Apprentice soon became a ,cultural phenomenon' among viewers in the key 18-49 age category. ,You're fired! ' became such a catch phrase that Trump even tried to trademark it.“

${ }^{29}$ Ansatzweise wird Trumps obszöne Ausstellung seines Reichtums allerdings in seinem (nachweislich nicht von ihm selbst verfassten) Buch The Art of the Deal konterkariert. So wird hier bereits im allerersten Absatz der banalen Logik des Tauschwerts eine Ästhetisierung des dealmaking (als Lifestyle und Praxis) gegenübergestellt. Vgl. Trump (2016: 1): „I don’t do it for the money. I've got enough, much more than I'll ever need. I do it to do it. Deals are my art form. Other people paint beautifully on canvas or write wonderful poetry. I like making deals, preferably big deals. That's how I get my kicks.“
} 
des Cool Capitalism mit einem spezifischen downdressing korrespondiert, verkörpert Trump, mit Žižek gesprochen, das tautologische Faktum einer ,unbeweglichen, obszönen, abstoßenden Präsenz" (Žižek 1991: 101).

In anderer Hinsicht lässt sich gleichwohl auch der von Trump verkörperte ,Uncool Capitalism“ mit dem neuen Geist des Kapitalismus in Verbindung bringen. Denn auch Trump geht es um die Positionierung als anti-elitär und anti-establishment: als charismatischer Outsider, der working Americans gegen Karrieristen, Bürokraten und Globalisten in Schutz nimmt. Wenn auf Trumps rallies auf ritualisierte Weise immer wieder der Rolling-Stones-Song „You Can't Always Get What You Want“ (1968) gespielt wird, dann kommt es auch hier zu einer gewissen Appropriation der Gegenkultur, d. h. der affektiven Aufladung seiner base mit den kulturellen Zeichen der Rebellion und des Protests. Dass ausgerechnet Trump die Empfindsamkeiten der weißen Unterschicht trifft, wenn er career politicians und the Washington establishment attackiert, lässt ihn gleichsam als bizarre Personifikation des von Hannah Arendt analysierten Bündnisses „Zwischen Mob und Elite“ (Arendt 1998: 702-725) erscheinen: Trump redet zu sehr so, wie viele seiner unterprivilegierten Anhänger*innen selber gerne reden würden, als dass sie ihn als Repräsentanten der Oberschicht (und ihrer ökonomischen Interessen) erkennen könnten. Dass diese Logik aufgeht, ist zweifellos einem umfassenden Prozess der ,Kulturalisierung ' geschuldet, durch den die Frage der Klassenzugehörigkeit stets kulturell überschrieben wird (vgl. Frank 2005; Michaels 2006).$^{30}$ Dies sollte freilich nicht darüber hinwegtäuschen, dass sich Trumps ,Uncool Capitalism“ weiterhin als Kapitalismus manifestiert, und dass sich der Bruch mit einigen Aspekten der neoliberalen Doktrin (Freihandel, Globalisierung) gut mit den Kontinuitäten in anderer Hinsicht (Deregulierung, Steuerpolitik, Plünderung des Sozialstaats) verträgt. Die Kompensation, die Trumps Unterstützer*innen aus der weißen Unterschicht erwarten können, ist daher auch nicht materieller, sondern rein affektiv-symbolischer Natur. Auch dies klingt im Soundtrack an, der auf Trumps elektrisierenden rallies läuft: „You Can't Always Get What You Want“ markiert jene Massenveranstaltungen insofern nicht nur als protestgeladen und gegenkulturell, sondern erinnert deren Teilnehmer*innen zugleich daran, was sie (,realistisch betrachtet ${ }^{\circ}$ ) erwarten und erhoffen dürfen - und was eben nicht.

\footnotetext{
${ }^{30}$ Mit Verweis auf Žižek lässt sich in diesem Zusammenhang argumentieren, dass es sich bei den andauernden amerikanischen culture wars (vgl. Hunter 1991) letztlich um einen, verschobenen Klassenkampf“ handelt. Vgl. Žižek (2009: 33): „Although the ,ruling class“ disagrees with the populists' moral agenda, it tolerates the ,moral war" as a means of keeping the lower classes in check, that is, it enables the latter to articulate their fury without disturbing the economic status quo. What this means is that the culture war is a class war in displaced mode pace those who claim we live in a post-class society."
} 


\section{Lechts/Rinks}

In einem einflussreichen Buch aus dem Jahr 2009 hat Mark Fisher sein Konzept des ,kapitalistischen Realismus ‘ formuliert. Was er darunter versteht, ist ,das weitverbreitete Gefühl, dass der Kapitalismus nicht nur das einzig gültige politische und ökonomische System darstellt, sondern dass es mittlerweile fast unmöglich geworden ist, sich eine kohärente Alternative dazu überhaupt vorzustellen" (Fisher 2013: 8). Anders gesagt: Die vielproklamierte ,Alternativlosigkeit“ der neoliberalen Hegemonie bleibt nicht auf die Sphären der Politik und der Ökonomie beschränkt, sondern macht auch vor Kultur, Subjektivität und Vorstellungskraft nicht halt. Als Beleg hierfür bezieht sich Fisher auf Beispiele aus der Populärkultur, so etwa auf das dystopische Genre des spätkapitalistischen Science-Fiction-Films, in dem sich Fredric Jamesons Diktum zu bestätigen scheint, dass es heute einfacher ist, ,sich das Ende der Welt vorzustellen als das Ende des Kapitalismus“ (ebd.: 8). ${ }^{31}$

Mit der Wahl Trumps ist es zwar weder leichter geworden, das Ende des Kapitalismus zu imaginieren, noch führt sein Bruch mit dem neoliberalen Status quo zu einer wirklichen Reaktivierung utopischer (d. h. nicht lediglich retrotopischer) „Impulse“ (Jameson 1988: 284). Dennoch kann es durchaus als blamabel empfunden werden, dass das Motto der linken Globalisierungskritik - ,Eine andere Welt ist möglich ${ }^{\text {- }}$ - heute scheinbar eher von rechts als von links beim Wort genommen wird. Gerade die parlamentarische Sozialdemokratie (Gerhard Schröders ,Neue Mitte ' oder Tony Blairs ,New Labour') und die Demokraten in den USA (Bill Clinton) hatten sich in den 1990er Jahren mit dem neoliberalen ,Ende der Geschichte“ arrangiert und viele der Deregulierungs-, Privatisierungsund Prekarisierungsinitiativen sogar eigenständig auf den Weg gebracht. Wenn heute auf beängstigende Weise von rechten Populisten demonstriert wird, dass eine Politik jenseits des post-politischen Konsenses in der Tat denkbar ist, dann sollte die Ursache für diese Entwicklung nicht zuletzt auch im Scheitern der Linken sowie in der allgemeinen Verkopplung von politischem Liberalismus und ökonomischem Neoliberalismus gesucht werden.

Indes wird deutlich, dass der Trump-Effekt bereits zu einer Reihe von ideologischen Verschiebungen und Rollenwechseln geführt hat. Insofern das Geschäft der Systemkritik heute in erster Linie von Rechtspopulisten betrieben wird, agiert die Linke zunehmend defensiv und gebiert sich als Verteidigerin der Institutionen und des alten Status quo. Diese Dynamik ist insbesondere in den USA zu beobachten, wo potenziell linke Themen von Trump, dem großen Disruptor, systematisch rechts überschrieben werden: Kritik am Irakkrieg, an Auslandseinsätzen des Militärs, an den Medien, den Geheimdiensten, am Freihandel, am

\footnotetext{
${ }^{31} \mathrm{Vgl}$. Jameson (1994: xii): „It seems to be easier for us today to imagine the thoroughgoing deterioration of the earth and of nature than the breakdown of late capitalism; perhaps that is due to some weakness in our imaginations." Zur ,dystopischen Imagination" im Kontext des Neoliberalismus, vgl. außerdem Schleusener (2017).
} 
,Globalismus'. Die meisten Demokraten dagegen stellen sich auf die Seite des FBI, verteidigen die NATO, NAFTA und andere Freihandelsabkommen oder bemängeln Trumps ,unamerikanische Rhetorik' (,This is not what America stands for!'). Ihr Optimismus beruht weniger auf der eigenen Programmatik als darauf, dass der Sonderermittler Robert Mueller - ein eingetragener Republikaner und von George W. Bush berufener ehemaliger FBI-Direktor - Trump und seinem Wahlkampfteam ,collusion with Russia' nachweisen wird. ${ }^{32}$ Die von Slavoj Žižek geäußerte Hoffnung, die Wahl Trumps könne einen Heilungsprozess innerhalb der Demokratischen Partei anstoßen (vgl. Žižek 2017), erweist sich vor diesem Hintergrund als frommer Wunsch. ${ }^{33}$

Was bei aller ideologischen Polarisierung im Trump-Amerika zum Ausdruck kommt, ist weniger die Proliferation neuer politischer Debatten und Diskurse als das Gefühl einer post-politischen Stasis. So verlaufen die Auseinandersetzungen zwischen den beiden Lagern nach einem routinierten Reiz-Reaktions-Schema, dessen talking points stets nur innerhalb der eigenen bubble Glaubwürdigkeit beanspruchen. Die Taktik, die kaum jemand so gut beherrscht wie Trump, besteht darin, den Gegner zu einer Reaktion zu zwingen, die diesen quasi selbst entlarvt. Etwa provoziert Trump die liberalen Medien durch seine permanenten Attacken und Invektiven dazu, den Schleier der Objektivität („CNN - The Most Trusted Name in News") abzustreifen und auf scheinbar einseitige Weise Trump-kritisch zu berichten. Der Effekt ist eine allgemeine Delegitimierung vermeintlich überparteilicher Institutionen, was jeden Maßstab tilgt, an dem sich die eigenen Proklamationen und Behauptungen noch messen lassen müssten. Wie noch zu zeigen sein wird, ist es nicht damit getan, die ,Lügen' zu entlarven, auf denen jene Politik des Post-Faktischen (vgl. Schleusener 2018b) beruht. Denn diese ist mit einer Affektökonomie verkoppelt, die - zumindest bis zu einem gewissem Grad - , an sich selbst ${ }^{\star}$ (d. h. an ihren unmittelbaren Effekten) zu beurteilen ist.

Der Eindruck des Statischen rührt zudem daher, dass die beiden Lager nicht nur aufeinander angewiesen sind, sondern sich auch vollständig zu neutralisieren scheinen. So antwortet auf jede liberale Kommentatorin, die ,Russian collusion!' ausruft, garantiert ein Trump-Anhänger mit dem Hinweis: ,But Hillary's emails...!" Auf diese Weise entsteht eine vollkommen zirkuläre Dynamik, eine Kreisbewegung, die sich aus sich selbst heraus anzutreiben scheint. Beispielsweise macht Trump eine herabsetzende Bemerkung über illegale Einwanderer (,hordes $^{6}$, ,rapists ${ }^{6}$ ); die Demokraten und die liberalen Medien stürzen sich darauf und

\footnotetext{
${ }^{32}$ Nachtrag April 2019: Mittlerweile hat Mueller seinen Bericht vorgelegt und Trumps Wahlkampfteam vom Vorwurf der koordinierten Zusammenarbeit mit Russland weitgehend entlastet.

${ }^{33}$ Nachtrag Juli 2019: Auch wenn sich der linke Flügel der Demokraten seit der Zwischenwahl 2018 im Aufwind fühlt, sind die 'linkspopulistischen' Positionen von Abgeordneten wie Alexandria Ocasio-Cortez in der Partei noch weitgehend marginalisiert. Dementsprechend gilt der moderate Joe Biden - und nicht etwa der erneut kandidierende Bernie Sanders - als wahrscheinlichster Herausforderer Trumps bei der nächsten Präsidentschaftswahl.
} 
werfen Trump Rassismus vor; Trump erklärt, er habe keinesfalls per se von Einwanderern gesprochen (,who are fine people'), sondern lediglich von potenziell gewalttätigen, illegalen Migrant*innen; die Demokraten entgegnen daraufhin, dass Trumps Xenophobie durch zahlreiche Beispiele belegt sei, und dass sein autokratisches Verhalten gegenüber den rechtsstaatlichen Institutionen beweise, dass es ihm gar nicht um das Problem der Legalität oder Illegalität gehe; woraufhin Trumps Anhänger*innen antworten, dass man sich in der Frage der Rechtsstaatlichkeit nicht von den Demokraten belehren lasse, da deren Akzeptanz illegaler Einwanderung hinlänglich zeige, dass ihre open door policies Rechtsstaatlichkeit ganz und gar ausschlössen. Und so weiter, ad infinitum...

Die Logik einer solchen Auseinandersetzung ist nicht in erster Linie auf das bessere Argument gemünzt, sondern eher darauf, den politischen Gegner der Illegitimität oder des Verrats zu überführen. Genau hierauf zielte schon der birtherism ab, d. h. die (nicht zuletzt auch von Trump lancierte) verschwörungstheoretische Behauptung, Barack Obama sei kein amerikanischer Staatsbürger, sondern ein in Kenia geborener Muslim. Das liberale Spiegelbild dieser Behauptung manifestiert sich heute in dem Vorwurf, Donald Trump sei durch die Hilfe Putins an die Macht gelangt und sei somit quasi ein ,russischer Agent". Im letzten Rededuell zwischen Trump und Hillary Clinton kam diese Logik der gegenseitigen Delegitimierung in einem denkwürdigen Schlagabtausch zum Ausdruck: Clintons Vorwurf an Trump, er sei eine Marionette Russlands (Putin „,would rather have a puppet as president of the United States“), pariert dieser, indem er ausruft: „No puppet. No puppet. You're the puppet!“ (vgl. Blake 2016).

Was sich hier letztlich artikuliert, ist im Falle beider Lager eine zwar unterschiedliche, aber gleichermaßen , organische“ Konzeption Amerikas. So basieren die politischen Logiken sowohl der Republikaner als auch der Demokraten auf einer je verschiedenen Variante des ,echten Amerikas', die mit einer jeweils anderen Form von Exzeptionalismus korrespondiert (vgl. Pease 2009). Folglich kommt es zur Bezichtigung der politischen Gegner*innen, die Werte und Grundsätze dieser als fundamental begriffenen ,imaginierten Gemeinschaft ${ }^{\star} \mathrm{zu}$ missachten oder $\mathrm{zu}$ verraten, das organische Gleichgewicht (die nativistische Vision eines Amerikas unter WASP-Vorherrschaft oder das multikulturelle Ideal der USA als nation of all nations) aus der Balance zu bringen. In gewisser Weise erinnert diese Konstellation an Deleuzes Beschreibung des klassischen amerikanischen Films, dessen Verklammerung von Kinematografie und Nationalmythologie durch den Begriff der ,organischen Repräsentation' markiert wird:

„Ein starkes ethisches Urteil muß die Ungerechtigkeit der ,Dinge“ anprangern, das Mitleid erregen und von der heraufkommenden neuen Zivilisation künden, kurzum: immer wieder Amerika entdecken...um so mehr, als von Anfang an strikt darauf verzichtet worden ist, nach den Ursachen zu forschen. Der amerikanische Film begnügt sich damit, die Erschlaffung einer Zivilisation (auf der Ebene des Milieus) und das Eingreifen eines Verräters (auf der Ebene der Handlung) anzuführen“ (Deleuze 1989: 206).

Vielleicht ist es kein Zufall, dass der vorliegende Aufsatz mit einer Parallele zwischen Trumps Schockpolitik und der (quasi avantgardistischen) Kinematografie des 
Zeit-Bilds begann, nun jedoch eine Analogie zum Bewegungs-Bild und den generischen Codes des konventionellsten amerikanischen Kinos aufzeigt. Denn der Trumpismus ist ganz wesentlich eine Hybridkonstruktion, deren affektive Ausdrucksform in deutlicher Diskrepanz steht zu seiner ideologischen Inhaltsform. Anders gesagt: Im Herzen populistischer Schockpolitik verbirgt sich letztlich ein retrotopisches, national-nativistisches Idyll. ${ }^{34}$ So korrespondiert auch der Schockfaktor von Trumps Mauer (ihre Durchstreichung demokratischer Offenheit und Gastfreundschaft) mit der idyllischen Logik des Schutzwalls als natürlicher Grenze zwischen innen und außen. Mit dem Bau dieser Mauer wird Amerika aller Sorgen ledig sein...

\section{Populistische Affekte}

Eines der Ziele des vorliegenden Aufsatzes bestand darin, die aktuelle ,Krise der Demokratie - die sich in kaum einem Ereignis so anschaulich manifestiert wie in der Wahl Trumps zum amerikanischen Präsidenten - nicht allein dem Aufstieg des Rechtspopulismus zuzuschreiben, sondern diesen stattdessen als symptomatisch für eine tiefer gehende Krise zu verstehen, die sich mit Begriffen wie ,Postdemokratie" (Crouch 2008), ,neoliberale Hegemonie" (Mouffe 2018) oder ,Politik der Alternativlosigkeit' (Fisher 2013) benennen lässt. Dass sich der in ganz Europa und den USA spürbare Rechtsruck zu einer Zeit ereignet hat, die von postdemokratischen Tendenzen, den allzu offensichtlich gewordenen Diskrepanzen der Globalisierung und der schwersten Wirtschafts- und Finanzkrise seit den 1920er und 1930er Jahren geprägt war, sollte nicht als zufällig begriffen werden. Vor diesem Hintergrund scheint es nicht abwegig, als ursächlich für den Aufschwung rechtspopulistischer Parteien und Bewegungen auch das tendenzielle Scheitern der Linken zu sehen, der es offensichtlich nicht gelungen ist, die verbreitete Unzufriedenheit mit dem neoliberalen Status quo und dem postdemokratischen Konsens für ,emanzipatorische' Zwecke nutzbar zu machen. Diese Analyse ist freilich nicht neu - und das Walter Benjamin zugeschriebene Bonmot, ,,dass jeder Aufstieg des Faschismus von einer gescheiterten Revolution zeugt“ (Žižek 2015: 14), ist wohl selten so oft zitiert worden wie in den letzten Jahren. ${ }^{35}$

Die Richtung dieses Arguments für glaubwürdig zu halten, sollte gleichwohl nicht zu einer allzu vereinfachenden Komplexitätsreduktion führen. Genauer gesagt: Die ökonomische Dimension der Krise der Demokratie zu betonen, heißt nicht zwangsläufig, einen simplen ,Ökonomismus` zu vertreten. So ist Trump keinesfalls allein von Wähler*innen aus der Arbeiter*innenklasse oder der Unterschicht gewählt worden, sondern - relativ gleich verteilt - von Amerikaner*innen aus sämtlichen

\footnotetext{
${ }^{34} \mathrm{Vgl}$. in diesem Zusammenhang auch den Begriff des Heartland, der auf die Arbeiten des Populismusforschers Paul Taggart zurïckgeht (Taggart 2000).

${ }^{35}$ Die Quelle des vermeintlichen Zitats ist umstritten. Überlegungen, die zumindest in die gedankliche Richtung des Diktums gehen, finden sich in den Texten „Über den Begriff der Geschichte“ und „Theorien des deutschen Faschismus“ (vgl. Benjamin 1991a und 1991b).
} 
Einkommensschichten. Was die Klassendimension indes relevant macht, ist die Tatsache, dass sich die ausschlaggebendsten Verschiebungen im Wahlverhalten in den unteren Einkommensgruppen ereignet haben, wo es zu massiven Zugewinnen im Lager von Trump und den Republikanern kam. Es stellt sich folglich die Frage, weshalb Wähler*innen, die sich als left out oder forgotten begreifen, die nicht von tax cuts for the rich oder der Abwicklung von Obamacare profitieren, in so großer Zahl für Trump gestimmt haben. Was konstituiert Trumps Attraktivität in dieser Konstellation? Und welche Mechanismen der politischen Imagination ermöglichen diese Dynamik?

Vielleicht sollte der Begriff der Ideologie hier nicht allzu pauschal verworfen werden. So erwähnt Arlie Russell Hochschild z. B. das ,große Paradox ‘ (Hochschild 2016: 1-82), dass der Widerstand gegen Bundeshilfen, staatliche Sozialprogramme und ökonomische Umverteilung genau dort am größten ist, wo diese Maßnahmen am nötigsten wären - etwa in den verarmten Staaten des amerikanischen Südens, die ausnahmslos in der Hand der Republikaner sind. Ohne Kenntnisnahme der systematisch betriebenen ökonomischen Desinformation in konservativen Milieus und durch rechte Medien (Fox News, talk radio usw.) lässt sich dieses Phänomen kaum adäquat erfassen (vgl. Schleusener 2014: 321-324). Der Begriff der Ideologie greift allerdings zu kurz, wenn es nicht lediglich um die Entlarvung von Täuschungsmanövern gehen soll, sondern zugleich um das Verständnis von Operationen, die laut Deleuze und Guattari ,kein Verkennen, keine Mißverständnisse sind, sondern vollkommen reaktionäre unbewußte Besetzungen“ (Deleuze/Guattari 1997: 331). Was Trump diesbezüglich eher vermag als ideologisch-argumentativ zu ,überzeugen“, ist die Mobilisierung und Modulation der Affekte seiner Anhänger*innenschaft. ${ }^{36}$ Hierzu schreibt Hochschild:

„Trump is an ,emotions candidate'. More than any other presidential candidate in decades, Trump focuses on eliciting and praising emotional responses from his fans rather than on detailed policy prescriptions. His speeches - evoking dominance, bravado, clarity, national pride, and personal uplift - inspire an emotional transformation. [...] Not only does Trump evoke emotion, he makes an object of it, presenting it back to his fans as a sign of collective success“" (Hochschild 2016: 225).

Hochschild, die über einen Zeitraum von fünf Jahren Interviews mit konservativen Tea-Party-Anhänger*innen in Louisiana geführt hat, beschreibt in dem daraus resultierenden Buch ein unter den Befragten vorherrschendes Gefühl des Verlusts, der Entfremdung und des (ökonomischen wie kulturellen) Abgehängtseins. Ein wesentlicher Baustein dieser ideologisch-affektiven Formation ist die Tatsache, dass sich die Verbitterung hier weniger auf die ökonomische Elite als auf das liberale Establishment bezieht, von dem man sich $\mathrm{zu}$ Unrecht $\mathrm{zu}$ rückschrittlichen „rednecks“ (ebd.: 23) degradiert fühlt. Analog dazu, wie der von Hillary Clinton

\footnotetext{
${ }^{36} \mathrm{Zu}$ einer derartigen ,Politik des Affekts', vgl. Massumi (2010 und 2015), Schleusener (2014 und 2015: 198-210) sowie Małecki/Schleusener (2015). Zu den Interferenzen von Affekt und Ideologie, vgl. John Protevis Konzeption einer ,affective ideology“ (Protevi 2016 und Peters/Protevi 2017).
} 
lancierte Begriff der ,deplorables ‘ von Trump-Anhänger*innen - durch einen klassischen Akt postmoderner Resignifikation (vgl. Zak 2017) - in eine Auszeichnung verwandelt wurde, kommt es insbesondere auf Trumps rallies zu einer , affektiven Umwandlung'. Anstatt sich dafür zu schämen, dass man die vom Establishment verordnete, politisch korrekte' Sprache nicht beherrscht, mündet Trumps öffentlich zur Schau gestellte Überschreitung der Grenzen politischer Korrektheit in ein nahezu kathartisches Gemeinschaftsgefühl - eine Art der ,kollektiven Efferveszenz', wie Hochschild in Anlehnung an Durkheim erklärt (Hochschild 2016: 225).

Trumps rallies sind in diesem Sinne mehr als simple Wahlkampfveranstaltungen, vielmehr sind sie Orte einer konkreten Retrotopie. Sachfragen und spezifische politische Vorschläge sind ebenso nebensächlich wie der Wahrheitswert von Trumps Parolen. Dass hier aber jemand öffentlich sagt, was man stets dachte, nicht sagen zu dürfen, ist die Grundlage von Trumps affektiver Ökonomie. Anstatt zu materieller Kompensation, für deren reelle Einforderung man über kein geeignetes Instrumentarium verfügt, kommt es immerhin zu einer Art von ,emotionaler Kompensation': einer affektiven Umverteilung in der unmittelbaren Gegenwart. Diese performativ operierende Affektmaschine - und ihre Umwandlung von Scham in Stolz, von Neid in Spott, von Angst vor sozialem Abstieg in Hass auf illegale Einwanderer - sollte freilich nicht als apolitisch oder unschuldig begriffen werden. Sie korrespondiert mit einem Programm, das eine ,Politik der Angst" (vgl. Woodward 2018) mit der Aussicht auf grenzenloses Wirtschaftswachstum verkoppelt - eine Konstellation, deren innere Diskrepanzen sich als ,affektive Dissonanz ${ }^{\circ}$ bemerkbar machen. ${ }^{37}$ So überlagert sich die hymnische Verkündung von ,jobs, jobs, jobs" mit einer nahezu dystopischen Panik bezüglich ,illegal aliens', , sanctuary cities', ,drug cartels', ,the deep state" und ,the fake news media'. Es mag sich die Frage stellen, ob der Affekt hier noch als Teilaspekt der politischen Auseinandersetzung fungiert, oder nicht vielmehr als deren Ersatz: als Fortsetzung der Politik mit anderen Mitteln.

\footnotetext{
${ }^{37}$,Affektive Dissonanz' meint hier die Überlagerung von im spinozistischen Sinne limitierenden Affekten (wie Trauer oder Angst) und mobilisierenden Affekten (wie Freude oder Hoffnung). Es lässt sich argumentieren, dass dieses Phänomen nicht nur für die Affektpolitik Trumps charakteristisch ist, sondern generell für jede gesellschaftlich-politische Formation, die sowohl von den Ansprüchen einer kapitalistischen Ökonomie als auch dem Kontrollregime des national security state geprägt ist. Mit Blick auf 9/11 und die Rolle der Medien, vgl. hierzu Massumi (2010: 54): „Die Medien boten keine Informationen oder Analyse. Es war die reine Affektmodulation [...]. Die ständigen Sicherheitsbedenken schleichen sich auf solch einer grundlegenden und gewohnheitsmäßigen Ebene in unser Leben ein, dass man sich kaum bewusst ist, wie sehr dadurch der generelle Tenor des täglichen Lebens verändert wird. Ganz ,instinktiv` schränkt man den eigenen Bewegungsrahmen und den Kontakt zu anderen Menschen ein. [...] Zur gleichen Zeit wie die Medien daran arbeiten, diese affektive Beschränkung herzustellen, helfen sie auf gewisse Weise auch, diese zu überwinden. Die Beschränkung darf nicht zu tiefgreifend sein, da ansonsten die Dynamik des Kapitalismus ausgebremst werden würde. Eine der größten Befürchtungen nach dem 11. September war, dass es aufgrund einer Krise im Verbrauchervertrauen zu einer wirtschaftlichen Rezession kommen würde. Somit wurde dazu aufgerufen, dass jeder als stolzer, patriotischer Akt weiter Geld ausgeben sollte.“
} 


\section{Literatur}

Agamben, Giorgio/Badiou, Alain/Bensaïd, Daniel/Brown, Wendy/Nancy, Jean-Luc/Rancière, Jacques/Ross, Kristin/Žižek, Slavoj: Demokratie? Eine Debatte. Berlin 2012.

Agerholm, Harriet: What is ,whitelash', and why are experts saying it led to Donald Trump's election? In: The Independent (09.11.2016), https://www.independent.co.uk/news/world/americas/us-elections/whitelash-what-is-it-white-vote-president-donald-trump-wins-us-election2016-a7407116.html (30.10.2018).

Arendt, Hannah: Elemente und Ursprünge totaler Herrschaft. Antisemitismus, Imperialismus, Totalitarismus. München 1998.

Avanessian, Armen/Hennig, Anke: Wer hat Angst nach dem Zeitgenössischen? In: Endlose Gegenwart. Donaufestival-Reader 2018: 166-175.

Bauman, Zygmunt: Retrotopia. Berlin 2017.

Benjamin, Walter: Über den Begriff der Geschichte. In: Ders.: Abhandlungen. Gesammelte Schriften Bd. 1.2. Frankfurt a. M. 1991a: 691-704.

Benjamin, Walter: Theorien des deutschen Faschismus. Zu der Sammelschrift ,Krieg und Krieger‘. Herausgegeben von Ernst Jünger. In: Ders.: Kritiken und Rezensionen. Gesammelte Schriften Bd. 3. Frankfurt a. M. 1991b: 238-250.

Best, Stephen/Marcus, Sharon: Surface Reading: An Introduction. In: Representations 108/1 (2009): 1-21.

Blake, Aaron: The final Trump-Clinton debate transcript, annotated. In: The Washington Post (19.10.2016), https://www.washingtonpost.com/news/the-fix/wp/2016/10/19/the-final-trump-clinton-debate-transcript-annotated/?utm_term=.c9c9d9cd90cf (30.10.2018).

Boltanki, Luc/Chiapello, Ève: Der neue Geist des Kapitalismus. Konstanz 2006.

Cowls, Josh/Schroeder, Ralph: Tweeting all the way to the White House. In: Pablo J. Boczkowski/Zizi Papacharissi (Hg.): Trump and the Media. Cambridge, Mass./London 2018: 151-157.

Crouch, Colin: Postdemokratie. Frankfurt a. M. 2008.

Davies, William: The Limits of Neoliberalism: Authority, Sovereignty and the Logic of Competition. Los Angeles/London 2017.

Davis, Stephen/Lukomnik, Jon/Pitt-Watson, David: The New Capitalists: How Citizen Investors Are Reshaping the Corporate Agenda. Boston 2006.

Deleuze, Gilles: Das Bewegungs-Bild. Kino 1. Frankfurt a. M. 1989.

Deleuze, Gilles: Das Zeit-Bild. Kino 2. Frankfurt a. M. 1997a.

Deleuze, Gilles: Differenz und Wiederholung. München 1997b.

Deleuze, Gilles/Guattari, Félix: Anti-Ödipus. Kapitalismus und Schizophrenie 1. Frankfurt a. M. 1997.

Duménil, Gérard/Lévy, Dominique: The Crisis of Neoliberalism. Cambridge/London 2011.

Fisher, Mark: Capitalist Realism: Is There No Alternative? Winchester 2009.

Fisher, Mark: Kapitalistischer Realismus ohne Alternative? Eine Flugschrift. Hamburg 2013.

Frank, Thomas: The Conquest of Cool: Business Culture, Counterculture, and the Rise of Hip Consumerism. Chicago 1998.

Frank, Thomas: What's the Matter with Kansas? How Conservatives Won the Heart of America. New York 2005.

Fraser, Nancy/Honneth, Axel: Umverteilung oder Anerkennung? Eine politisch-philosophische Kontroverse. Frankfurt a. M. 2003.

Greenberg, Jonathan: Trump lied to me about his wealth to get onto the Forbes 400. Here are the tapes. In: The Washington Post (20.04.2018), https://www.washingtonpost.com/outlook/ trump-lied-to-me-about-his-wealth-to-get-onto-the-forbes-400-here-are-the-tapes/2018/04/20/ ac762b08-4287-11e8-8569-26fda6b404c7_story.html?noredirect $=$ on\&utm_term $=$. cdd9ble171da (30.10.2018).

Hagen, Wolfgang: Gegenwartsvergessenheit: Lazarsfeld -Adorno - Innis - Luhmann. Berlin 2003.

Haider, Asad: Mistaken Identity: Race and Class in the Age of Trump. London/New York 2018.

Henley, Jon: White and wealthy voters gave victory to Donald Trump, exit polls show. In: The Guardian (09.11.2016), https://www.theguardian.com/us-news/2016/nov/09/white-voters-victory-donald-trump-exit-polls (30.10.2018). 
Hochschild, Arlie Russell: Strangers in Their Own Land: Anger and Mourning on the American Right. New York 2016.

Holmes, Christopher: Polanyi in Times of Populism: Vision and Contradiction in the History of Economic Ideas. New York 2018.

Horn, Eva: Zukunft als Katastrophe. Frankfurt a. M. 2014.

Hunter, James Davison: Culture Wars: The Struggle to Define America. New York 1991.

Jameson, Fredric: Das politische Unbewußte. Literatur als Symbol sozialen Handelns. Reinbek bei Hamburg 1988.

Jameson, Fredric: Postmodernism, or, The Cultural Logic of Late Capitalism. London/New York 1992.

Jameson, Fredric: The Seeds of Time. New York 1994.

Jörke, Dirk/Selk, Veith: Theorien des Populismus. Eine Einführung. Hamburg 2017.

Keefe, Patrick Radden: How Mark Burnett resurrected Donald Trump as an icon of American success. In: The New Yorker (07.01.2019), https://www.newyorker.com/magazine/2019/01/07/ how-mark-burnett-resurrected-donald-trump-as-an-icon-of-american-success (28.12.2018).

King Jr., Neil: Trump on 2011: ,Part of beauty of me is I'm very rich'. In: The Wall Street Journal - Washington Wire (17.03.2011), https://blogs.wsj.com/washwire/2011/03/17/trump-on2012-part-of-beauty-of-me-is-im-very-rich/ (30.10.2018).

Klein, Naomi: No Is Not Enough: Defeating the New Shock Politics. London 2017.

Koch, Lars: Walling out. Zur Diskurspolitik und Mythomotorik Neuer Mauern in der Populärkultur. In: Johannes Becker/Benjamin Bühler/Sandra Pravica/Stefan Willer (Hg.): Zukunftssicherung. Kulturwissenschaftiche Perspektiven. Bielefeld 2019: 167-184.

Koch, Lars/Nanz, Tobias: Ästhetische Experimente. Zur Ereignishaftigkeit und Funktion von Störungen in den Künsten. In: Zeitschrift für Literaturwissenschaft und Linguistik, 173 (2014): 94-115.

Koch, Lars/Nanz, Tobias/Pause, Johannes (Hg.): Imaginationen der Störung. Behemoth. A Journal on Civilisation, 9/1 (2016).

Lammert, Christian/Vormann, Boris: Die Krise der Demokratie und wie wir sie überwinden. Berlin 2017.

Małecki, Wojciech/Schleusener, Simon: ,What affects are you capable of?' On Deleuze and somaesthetics. In: Simone Bignall/Sean Bowden/Paul Patton (Hg.): Deleuze and Pragmatism. London/New York 2015: 216-234.

Manow, Philip: Die politische Ökonomie des Populismus. Berlin 2018.

Mason, Paul: PostCapitalism: A Guide to Our Future. London 2015.

Massumi, Brian: Ontomacht: Kunst, Affekt und das Ereignis des Politischen. Berlin 2010.

Massumi, Brian: Politics of Affect. Cambridge, UK/Malden 2015.

McGuigan, Jim: Cool Capitalism. London/New York 2009.

Mehta, Seema: Transcript: Clinton's full remarks as she called half of Trump supporters ,deplorables'. In: Los Angeles Times (10.09.2016), https://www.latimes.com/nation/politics/trailguide/la-na-trailguide-updates-transcript-clinton-s-full-remarks-as-1473549076htmlstory.html (28.10.2018).

Michaels, Walter Benn: The Trouble with Diversity. How We Learned to Love Identity and Ignore Inequality. New York 2006.

Mouffe, Chantal: On the Political. London 2005.

Mouffe, Chantal: For a Left Populism. New York/London 2018.

Pease, Donald: The New American Exceptionalism. Minneapolis/London 2009.

Peters, Christian Helge/Protevi, John: Affective ideology and Trump's popularity (draft paper, 28.09.2017), http://www.protevi.com/john/TrumpAffect.pdf (30.10.2018).

Polanyi, Karl: The Great Transformation: The Political and Economic Origins of Our Time [1944]. Boston 2001.

Protevi, John: Stanley on ideology. In: Theoria 31/3 (2016): 357-369.

RealClearPolitics: President Trump job approval, https://www.realclearpolitics.com/epolls/other/ president_trump_job_approval-6179.html (30.10.2018).

Schleusener, Simon: Neoliberal affects: The cultural logic of cool capitalism. In: REAL: Yearbook of Research in English and American Literature 30 (2014): 307-326.

Schleusener, Simon: Kulturelle Komplexität: Gilles Deleuze und die Kulturtheorie der American Studies. Bielefeld 2015. 
Schleusener, Simon: The dialectics of mobility: Capitalism and apocalypse in Cormac McCarthy's The Road. In: European Journal of American Studies 12/3 (2017): 1-14.

Schleusener, Simon: Political disconnects: Donald Trump, the cultural left, and the crisis of neoliberalism. In: Coils of the Serpent: Journal for the Study of Contemporary Power 2 (2018a): $20-34$.

Schleusener, Simon: Post-truth politics: The new right and the postmodern legacy. In: Ilka Brasch/Ruth Mayer (Hg.): Modernities and Modernization in North America. Heidelberg 2018b: 353-370.

Schorr, Daniel: A new, ,post-racial' political era in America (Transcript). In: NPR (28.01.2008), https://www.npr.org/templates/story/story.php?storyId=18489466\&t=1540947603093 (30.10.2018).

Seeßlen, Georg: Trump! Populismus als Politik. Berlin 2017.

Stegemann, Bernd: Das Gespenst des Populismus. Ein Essay zur politischen Dramaturgie. Berlin 2017.

Streeck, Wolfgang: How Will Capitalism End? Essays on a Failing System. London/New York 2016.

Strick, Simon: Alt-Right-Affekte. Provokationen und Online-Taktiken. In: Zeitschrift für Medienwissenschaft 19/2 (2018): 113-125.

Taggart, Paul: Populism. Buckingham/Philadelphia 2000.

Taylor, Charles: Multiculturalism and the Politics of Recognition: An Essay. Princeton 1992.

Trump, Donald (\& Tony Schwartz): The Art of the Deal [1987]. London 2016.

Trump Twitter Archive, http://www.trumptwitterarchive.com (21.12.2018).

Turner, Fred: Trump on twitter: How a medium designed for democracy became an authoritarian's mouthpiece. In: Pablo J. Boczkowski/Zizi Papacharissi (Hg.): Trump and the Media. Cambridge, Mass./London 2018: 143-149.

Virilio, Paul: Geschwindigkeit und Politik. Berlin 1980.

Virilio, Paul: Ästhetik des Verschwindens. Berlin 1986.

Virilio, Paul: Revolutionen der Geschwindigkeit. Berlin 1993.

Wallerstein, Immanuel/Collins, Randall/Mann, Michael/Derluguian, Georgi/Calhoun, Craig: Does Capitalism Have a Future? Oxford/New York 2013.

Weber, Max: Die protestantische Ethik I. Eine Aufsatzsammlung. Hg. von J. Winckelmann. Gütersloh 1991.

Wolff, Michael: Fire and Fury: Inside the Trump White House. New York 2018.

Woodward, Ben: Fear: Trump in the White House. New York 2018.

Woolf, Nicky: ,Nasty woman': Trump attacks Clinton during final debate. In: The Guardian (20.10.2016), https://www.theguardian.com/us-news/2016/oct/20/nasty-woman-donald-trumphillary-clinton (30.10.2018).

Zak, Dan: Embracing the insult, disarming the enemy. In: The Washington Post (16.01.2017): C1.

Žižek, Slavoj: Liebe Dein Symptom wie Dich selbst! Jacques Lacans Psychoanalyse und die Medien. Berlin 1991.

Žižek, Slavoj: First as Tragedy, Then as Farce. London/New York 2009.

Žižek, Slavoj: Blasphemische Gedanken: Islam und Moderne. Berlin 2015.

Žižek, Slavoj: Slavoj Žižek on Trump and Brexit. In: BBC News (17.01.2017), https://www.youtube.com/watch?v=2ZUCemb2plE $(30.10 .2018)$.

\section{Abbildungen}

Abb. 1-4: Graphics from the article 'Election 2016: Exit Polls' by Jon Huang, Samuel Jacoby, Michael Strickland and K.K. Rebecca Lai originally appeared in the The New York Times on November 8, 2016 are copyright The New York Times and are used here by permission. Huang, Jon/Jacoby, Samuel/Strickland, Michael/Lai, K. K. Rebecca: Election 2016: Exit Polls. In: The New York Times (08.11.2016), https://www.nytimes.com/interactive/2016/11/08/us/politics/election-exit-polls.html (29.12.2018). 
Open Access Dieses Kapitel wird unter der Creative Commons Namensnennung 4.0 International Lizenz (http://creativecommons.org/licenses/by/4.0/deed.de) veröffentlicht, welche die Nutzung, Vervielfältigung, Bearbeitung, Verbreitung und Wiedergabe in jeglichem Medium und Format erlaubt, sofern Sie den/die ursprünglichen Autor(en) und die Quelle ordnungsgemäß nennen, einen Link zur Creative Commons Lizenz beifügen und angeben, ob Änderungen vorgenommen wurden.

Die in diesem Kapitel enthaltenen Bilder und sonstiges Drittmaterial unterliegen ebenfalls der genannten Creative Commons Lizenz, sofern sich aus der Abbildungslegende nichts anderes ergibt. Sofern das betreffende Material nicht unter der genannten Creative Commons Lizenz steht und die betreffende Handlung nicht nach gesetzlichen Vorschriften erlaubt ist, ist für die oben aufgeführten Weiterverwendungen des Materials die Einwilligung des jeweiligen Rechteinhabers einzuholen. 